\title{
Técnica quirúrgica de la traqueostomía percutánea en el paciente con COVID-19
}

\author{
Percutaneous tracheostomy surgical technique in the patient with \\ COVID-19
}

\author{
Fernando Rodríguez H. ${ }^{1}$, Jose J. Serna', Alberto F. García' ${ }^{1}$, María X. Revelo², \\ Mónica Bejarano ${ }^{3}$, Alexander Salcedo', Carlos A. Ordóñez \\ Médico, Especialista en Cirugía general y Cirugia de Trauma y Emergencias, Sección de Cirugia de Trauma y Emergencias, \\ Departamento de Cirugia, Fundación Valle del Lili. \\ 2 Enfermera, Centro de investigaciones Clínicas (CIC), Fundación Valle del Lili. Cali, Colombia. \\ 3 Médico, Especialista en Cirugía general, Magíster en Epidemiología, Sección de Cirugia de Trauma y Emergencias, Departamento \\ de Cirugia, Fundación Valle del Lili.
}

\section{Resumen}

Generalidades. Desde diciembre de 2019 se detectó una nueva infección respiratoria, causada por el virus denominado SARS-CoV-2, decretada posteriormente como pandemia, lo cual ha exigido al personal de salud replantear la forma de prestar sus servicios en salud y garantizar la auto-protección con recursos que han sido insuficientes incluso en los países más desarrollados.

Dado que la transmisión del SARS-CoV-2 ocurre a través de aerosoles expulsados de la vía aérea, que pueden ser inhalados o llevados a las mucosas por contacto con las manos contaminadas, se requiere minimizar la posibilidad de contagio para los equipos de atención en salud.

Objetivos. Brindar herramientas a los cirujanos que les permitan elegir la técnica con menor probabilidad de exposición a aerosoles. Describir el paso a paso de la técnica quirúrgica de la traqueostomía percutánea, enfatizando en el control sobre la generación de aerosoles en pasos críticos.

Aspectos técnicos. La técnica completamente percutánea con kit de traqueostomía permite un mejor sello entre tejidos y dispositivos. Los escenarios más frecuentes para realizar una traqueostomía son: el paciente intubado con ventilación mecánica y el paciente con falla en la intubación que requiere una intervención de emergencia.

Conclusión. El alto contagio del COVID-I9 al practicar intervenciones en la vía aérea nos obliga a hacer énfasis en las estrategias que reduzcan la formación de aerosoles y permitan la contención de los mismos durante la realización de traqueostomías.

Palabras clave: COVID-I9; virus del SRAS; coronavirus; infecciones por coronavirus; pandemias; cirugía general; traqueostomía.

Fecha de recibido: 11/04/2020 - Fecha de aceptación: 13/04/2020

Correspondencia: Fernando Rodriguez-Holguin. Dirección: Carrera 98 \# 18-49, Centro de investigaciones Clínicas (CIC), Fundación Valle del Lili. Cali, Colombia. Celular: 315-4112938

Correo electrónico: fernando.rodriguez.ho@fvl.org.co

Citar como: Rodríguez HF, Serna JJ, García AF, Revelo MX, Bejarano M, Salcedo A. Técnica quirúrgica de la traqueostomía percutánea en el paciente con COVID-19. Rev Colomb Cir. 2020;35:182-9 / Especial COVID -19. https://doi.org/10.30944/20117582.607

Este es un artículo de acceso abierto bajo una Licencia Creative Commons - BY-NC-ND https://creativecommons.org/licenses/by-ncnd/4.0/deed.es 


\begin{abstract}
Background: Since December 2019, a new respiratory infection was detected, caused by the virus called SARS$\mathrm{CoV}-2$, later decreed as a pandemic, which has required health personnel to rethink the way of providing their health services and guarantee the self-protection with resources that have been insufficient even in the most developed countries. As the transmission of SARS-CoV-2 occurs through aerosols expelled from the airway, which can be inhaled or brought to the mucosa by contact with contaminated hands, it is necessary to minimize the possibility of contagion for the health care teams.

Objectives: Provide tools to surgeons that allow them to choose the technique with the least probability of exposure to aerosols. Describe the step-by-step of the percutaneous tracheostomy technique, emphasizing control about the generation of aerosols in critical steps.

Technical aspects: The fully percutaneous technique with a tracheostomy kit allows a better seal between tissues and devices. The most frequent scenarios for performing a tracheostomy are an intubated patient with mechanical ventilation and a patient with failure of intubation that requires emergency intervention.

Conclusion: The high contagion of COVID-I9 when practicing airway interventions forces us to emphasize strategies that reduce the formation of aerosols and allow them to be contained during tracheostomies.
\end{abstract}

Keywords: COVID-I9; SARS virus; coronavirus; coronavirus infections; pandemics; general surgery; tracheostomy.

\section{Introducción}

En Diciembre de 2019 la humanidad comenzó a enfrentarse a una nueva enfermedad infecciosa, un tipo de neumonía que emergió en la ciudad de Wuhan, China. Para final del mes, se dio aviso a la Organización Mundial de la Salud (OMS), que inicialmente consideró la enfermedad "prevenible y controlable". En la primera semana de enero de este año, ya se tenía conocimiento que ésta enfermedad era causada por un nuevo coronavirus, similar a otros que producen resfriados estacionales en humanos ${ }^{\mathrm{I}}$.

Esta infección que se diseminó inicialmente por países de oriente, rápidamente llegó a Europa y luego a Estados Unidos, aumentando dramáticamente el número de pacientes críticos con necesidad de permanecer en unidades de cuidados intensivos y ocasionando un número importante de muertes, por lo que el 30 de enero la OMS declara la "Emergencia Global" y el II de marzo ya es considerada como pandemia ${ }^{\mathrm{I}}$.

La afectación de forma masiva a países en los cinco continentes, ha desbordado los sistemas de salud de países desarrollados y amenaza con arrasar de la misma manera con los limitados recursos de los sistemas de salud de países más pobres y en vía de desarrollo, como es el caso de varios países de Latinoamérica.

Como personal de salud nos vemos afectados especialmente por esta pandemia, porque somos parte de una especie que está siendo diezmada por una enfermedad invisible que en pocas semanas nos ha obligado a cambiar nuestra forma de vivir, y por otro lado, porque como personal sanitario debemos hacer frente al desastre y desarrollar las estrategias para defender a nuestros congéneres, al tiempo que nos protegemos y evitamos ser infectados por el virus.

\section{Generalidades}

La principal fuente de transmisión del SARSCoV-2 son los aerosoles de la vía aérea, con los cuales podemos hacer contacto en dos momentos específicos:

- Inhalando partículas expulsadas en forma de aerosol cuando tenemos un contacto estrecho y cercano (menos de I,5 metros) por más de I5 minutos con un paciente con COVID-I9. 
- A través de las mucosas, a partir de un contacto inicial con superficies contaminadas por aerosoles, que fácilmente son transportadas por nuestras manos hacia las mucosas, por donde ingresa el virus.

Estas dos condiciones se presentan en el momento de practicar procedimientos, especialmente aquellos que tienen relación con la vía aérea y han sido denominados "Procedimientos Generadores de Aerosoles" (PGA) ${ }^{2}$. Es así como la intubación orotraqueal y el manejo quirúrgico de la vía aérea merecen un especial cuidado para evitar el contagio del equipo de salud, por el alto riesgo de diseminación de partículas de aerosoles que contienen el virus ${ }^{3}$. Esta situación se complica aún más cuando el manejo de la vía aérea incluye procedimientos de urgencia en escenarios de riesgo de muerte del paciente.

Es importante tener en cuenta que la seguridad del equipo sanitario siempre debe ser una prioridad, más aun en los escenarios en los cuales las necesidades en la atención de pacientes exceden los recursos disponibles, situación que se evidencia cada vez con más frecuencia en la actual pandemia. Por ello es indispensable hacer énfasis en el uso de todos los elementos de protección personal (EPP) requeridos para la atención de los pacientes con COVID-I9 confirmada o sospechosa, incluso en los escenarios de urgencia más críticos ${ }^{2,4}$.

Lo anterior no significa que debemos sacrificar la vida de nuestro paciente para resguardar nuestra seguridad y la de nuestro equipo, significa que debemos preveer, con anticipación, cualquier posible dificultad o complicación antes de embarcarnos en un procedimiento de este tipo, adelantándonos mediante el alistamiento de los recursos potencialmente útiles en un momento dado, basados en estrategias múltiples, secuenciales y planeadas por todo el grupo quirúrgico, que permitan actuar de forma coordinada y dentro de un plan trazado, antes de iniciar, y aunque el plan puede cambiar en el transcurso del acto quirúrgico, incluso ese cambio se debe canalizar dentro de un protocolo definido con anterioridad.

\section{Objetivos}

Más allá de discutir sobre las indicaciones de una traqueostomía, o el momento más apropiado para realizarla, este documento tiene como objetivo principal, brindar herramientas al cirujano encargado de practicar las traqueostomías, para tomar la decisión sobre la técnica quirúrgica ideal en los pacientes con diagnostico o sospecha de COVID-I9, teniendo en cuenta aspectos que en situaciones operativas usuales tienen menos relevancia.

Se analizaron aspectos de seguridad del equipo quirúrgico frente a la probabilidad de contagio en el procedimiento como prioridad, así como aspectos basados en la seguridad del paciente durante el procedimiento. Se describe la técnica propuesta paso a paso en dos situaciones: el paciente con vía aérea asegurada, intubado y con ventilación asistida y el paciente en quien se falla al intento de la intubación orotraqueal inicial, que requiere manejo quirúrgico de la vía aérea urgente.

\section{Aspectos técnicos}

Para la traqueostomía se conocen varias opciones: la técnica abierta, la técnica parcialmente percutánea y la técnica completamente percutánea. Consideramos que esta última ofrece el mejor control sobre la diseminación de aerosoles, ya que por el sellado que realizan los tejidos blandos alrededor de los implementos del Kit, se logra una mejor contención de los aerosoles, salvo en algunos momentos de intercambio de dispositivos durante la dilatación de los tejidos, lo que se puede controlar parcialmente con algunas estrategias que se describirán más adelantes.

Adicionalmente, como la mayoría de las traqueostomías se requieren en pacientes ventilados en UCI, esta técnica es muy favorable para la realización en la propia cama del paciente, de forma segura, en poco tiempo, sin requerir traslado a quirófanos. La técnica abierta por otro lado, requiere un poco más de tiempo quirúrgico ${ }^{6}$, y altera la ergonomía del cirujano cuando se realiza en la cama del paciente, pudiendo llegar a comprome- 
ter la seguridad del paciente al tener que operar en posiciones incómodas para el cirujano; además, requiere de dispositivos de electrocauterio sobre la vía aérea, lo que aumenta la probabilidad de vaporización de virus alojado en la vía aérea ${ }^{3}$.

Si bien, en la técnica percutánea ocurre una descompresión de la vía aérea que puede favorecer la formación de aerosoles, esto se puede mitigar fácilmente con dos medidas: relajación completa del paciente para que no tenga esfuerzo ventilatorio ni tos, y suspensión de la ventilación en espiración antes de desinflar el neumotaponador, hacer la punción y dilatar la tráquea4

Somos conscientes que de varios autores ${ }^{7}$ prefirieron la traqueostomía por vía abierta durante la epidemia de SARS y que otros describen que la técnica percutánea requiere el uso de fibrobroncoscopia con la creencia que agrega seguridad al procedimiento, lo que aumenta la manipulación de la vía aérea ${ }^{8}$, sin embargo, al comparar las diferentes técnicas con guía anatómica versus guía con broncoscopia, no se ha encontrado diferencias en el número de complicaciones, por lo que, sumado al mayor riesgo de contaminación y contagio durante la broncoscopia en los pacientes con COVID-I9 ${ }^{4}$, en Fundación Valle del Lili, en Cali, Colombia, preferimos la técnica percutánea con guía anatómica frente a la técnica abierta 9 .

En el primer escenario, de la traqueostomía completamente percutánea en un paciente previamente intubado, con vía aérea controlada y conectado a ventilación mecánica, es importante recordar que al tener la vía aérea asegurada, la traqueostomía no es una urgencia. Por consiguiente, debemos aprovechar la ventaja que esto nos confiere y siempre mantener control de la vía aérea, lo que empieza por elegir en conjunto con el equipo tratante de cuidados intensivos el mejor momento clínico para la práctica de este procedimiento, ya que si la traqueostomía no le va a significar una ventaja en el manejo o la recuperación al paciente y el riesgo que se corre no sea justificado por los beneficios que obtendrá el paciente, probablemente sea preferible aplazar el procedimiento hasta que el paciente ten- ga parámetros ventilatorios más favorables, que permitan llevar a cabo la traqueostomía en un paciente que tolere mejor los cambios de presión de la vía aérea sin comprometer la oxigenación, disminuyendo así las probabilidades de caer en una situación de urgencia que pueda comprometer el control del procedimiento9.

En el segundo caso, de la traqueostomía completamente percutánea en un paciente con falla en la intubación orotraqueal con requerimiento de manejo quirúrgico urgente de la vía aérea, si bien es un escenario menos controlado donde se corre el riesgo de perder la vida del paciente, se debe reconocer lo más pronto posible cuando una vía aérea será difícil, para evitar hacer múltiples intentos de intubación y cambiar rápidamente a estrategias de control de la vía aérea para proporcionar algo de ventilación y oxigenación, como transición mientras se logra la traqueostomía. Esto significa que se debe dar aviso tempranamente al equipo quirúrgico para alistarse con los EPP requeridos para estos casos ${ }^{9}$, evitando llegar a situaciones desesperadas de mayor descontrol que nos lleven a cometer errores que puedan afectar la seguridad del equipo. Siempre nuestro objetivo es llevar la situación de poco control, a una situación de mejor control, haciendo una pausa ante la primera falla de intubación, para repensar la estrategia antes de llegar a situaciones desesperadas, y plantear la posibilidad de uso temporal de máscaras laríngeas de última generación, que permitan oxigenar el paciente mientras se realiza una traqueostomía.

El otro objetivo a tener presente es evitar la realización de procedimientos quirúrgicos no definitivos como la cricotiroidotomía, ya que expone al equipo sanitario a dos PGA, puesto que después es necesario realizar la traqueostomía definitiva, teniendo que manejar el orificio de la cricotiroidotomía por donde se podrán generar fugas y por consiguiente aerosoles. Aunque sabemos que la cricotiroidotomía es un procedimiento salvador en casos de extrema urgencia, en pacientes con COVID-I9 es ideal tratar de realizar el procedimiento definitivo (traqueostomía) al primer intento. 


\section{Técnica quirúrgica}

\section{Paciente con vía aérea asegurada con TOT}

I. Verifique el diligenciamiento del consentimiento informado con probabilidad de muerte.

2. Realice chequeo de seguridad de uso completo de elementos de protección personal (EPP) de máximo nivel, antes de ingresar al cubículo del paciente.

3. Confirme que el paciente esté recibiendo oxígeno con $\mathrm{FIO}_{2}$ IOO\% al ingresar al cubículo.

4. Solicite y confirme capnografía.

5. Posicione al paciente con rollo interescapular.

6. Retire cables de monitoria del frente del paciente y páselos detrás de los hombros.

7. Retire la fijación del tubo orotraqueal (TOT), dejando inflado el neumotaponador.

8. Coloque una compresa en boca y nariz del paciente, conteniendo posibles partículas de aerosol generadas por fugas del TOT durante el procedimiento.

9. Haga asepsia según protocolo institucional.

Io. Coloque campos quirúrgicos.

II. Pausa de seguridad para verificar los elementos del Kit de traqueostomía e insumos completos, incluyendo cánula del tamaño adecuado en el campo quirúrgico.

I2. Infiltre piel con Lidocaína al $\mathbf{2} \%$.

I3. Confirme relajación completa del paciente.

I4. Fije la tráquea del paciente con su mano no dominante.

15. Puncione la piel insinuando la aguja en la tráquea hasta sentir el TOT.

I6. Avance la sonda de aspiración de sistema cerrado, a manera de guía para avanzar el TOT en caso de pérdida de la vía aérea.

17. Suspenda la ventilación mecánica en espiración completa y mantenga en apnea.

I8. Desinfle el neumotaponador y retire lentamente el TOT sin extubar.
19. Puncione la tráquea y verifique aspiración de aire.

20. Pase la guía metálica sin resistencia y retire la aguja.

2I. Haga incisión en piel de $5 \mathrm{~mm}$ con el bisturí del Kit.

22. Dilate con Rhino y cubra con compresa alrededor de éste antes de retirarlo para atrapar los aerosoles (el paciente está en apnea desde el paso I6).

23. Pase la cánula de traqueostomía, manteniendo protección de los bordes con la compresa.

24. Retire el dilatador, la guía y el conductor de la guía al tiempo que se cubre la luz de la cánula con la compresa.

25. Coloque la endocánula para ventilación (la del conector blanco en todas las casas medicas), cubriendo su luz con la compresa.

26. Infle el neumotaponador.

27. Conecte las mangueras de ventilación mecánica con capnografía.

28. Confirme capnografía.

29. Retire la sonda de aspiración que servía de guía.

30. Instale el sistema de fijación de la traqueostomía.

3I. Cubra los bordes del traqueostoma con gasas por ambos lados.

32. Retire el TOT clampeado con pinza Rochester, cubriendo boca y nariz del paciente con compresa para protección de posibles fugas generadoras de aerosoles.

Si el paciente presenta disminución en la saturación de oxígeno por debajo de $80 \%$ entre los pasos i8 al 22 y se considera necesaria y apropiada la ventilación de rescate, se debe dejar la guía metálica avanzada en la vía aérea, avanzar el TOT sobre la sonda de aspiración, lo suficiente para que el balón ocluya el orificio de la tráquea, e inflarlo en esta posición, para dar las ventila- 
ciones de rescate hasta que el paciente recupere su oxigenación. Una vez tenga la oximetría adecuada, se debe retomar el procedimiento en el paso en que se suspendió. Se debe mantener siempre cubierto el traqueostoma con compresa para evitar diseminación de aerosoles.

Si ya se ha dilatado de manera adecuada el traqueostoma cuando el paciente presenta disminución en la saturación de oxígeno y hay certeza de la posición dentro de vía aérea, se debe pasar la cánula de traqueostomía y completar el procedimiento como se ha indicado.

\section{Paciente urgente con falla en la intubación}

I. Realice chequeo de seguridad de uso completo de elementos de protección personal (EPP) de máximo nivel, antes de ingresar al quirófano o el cubículo del paciente.

2. Solicite ventilación de rescate a través de mascara laríngea con $\mathrm{FIO}_{2} \mathrm{IOO} \%$.

3. Solicite y confirme capnografía.

4. Posicione al paciente con rollo interescapular.

5. Retire cables de monitoria del frente del paciente y páselos detrás de los hombros.

6. Coloque una compresa en boca y nariz del paciente, conteniendo posibles partículas de aerosol generadas por fugas del TOT durante el procedimiento.

7. Haga asepsia según protocolo institucional.

8. Coloque campos quirúrgicos.

9. Pausa de seguridad para verificar los elementos del Kit de traqueostomía e insumos completos, incluyendo cánula del tamaño adecuado en el campo quirúrgico.

Io. Infiltre piel, plano subcutáneo y pretraqueal con Lidocaína al $2 \%$; deje un poco para la luz traqueal.

II. Confirme relajación completa del paciente, siempre y cuando se pueda mantener ventilación adecuada a través de la máscara laríngea.

I2. Fije la tráquea del paciente con su mano no dominante.
I3. Suspenda la ventilación mecánica en espiración completa y mantenga en apnea.

I4. Puncione la tráquea y verifique aspiración de aire.

I5. Pase la guía metálica sin resistencia y retire la aguja.

I6. Haga incisión en piel de $5 \mathrm{~mm}$ con el bisturí del Kit.

I7. Dilate con Rhino y cubra con compresa alrededor de éste antes de retirarlo para atrapar aerosoles (el paciente está en apnea desde el paso I3).

I8. Pase la cánula de traqueostomía, manteniendo protección de los bordes con la compresa.

19. Retire el dilatador, la guía y el conductor de guía, al tiempo que se cubre la luz de la cánula con la compresa.

20. Coloque la endocánula para ventilación (la del conector blanco en todas las casas medicas), cubriendo su luz con compresa.

2I. Infle el neumotaponador.

22. Conecte las mangueras de ventilación mecánica con capnografía.

23. Confirme la capnografía.

24. Instale el sistema de fijación de la traqueostomía.

25. Cubra los bordes del traqueostoma con gasas por ambos lados.

26. Retire la máscara laríngea clampeada con pinza Rochester, cubriendo boca y nariz del paciente con compresa para protección de posibles fugas generadoras de aerosoles.

Si el paciente presenta disminución en la saturación de oxígeno por debajo de $80 \%$ entre los pasos I 3 al I7 y se considera necesaria y apropiada la ventilación de rescate, se debe dejar la guía metálica avanzada en la vía aérea, retirar el dilatador Rhino dejando la guía con el conductor dentro de la tráquea, hacer oclusión digital del orificio en la tráquea evitando que se genere enfisema subcutáneo al reiniciar la ventilación y reiniciar ventilaciones de rescate hasta que el 
paciente recupere su oxigenación. Una vez se recupere la oximetría adecuada, se debe retomar el procedimiento en el paso en que se suspendió el procedimiento. Se debe mantener siempre cubierto el traqueostoma con compresa para evitar diseminación de aerosoles.

Si ya se ha dilatado de manera adecuada el traqueostoma cuando el paciente presenta disminución en la saturación de oxígeno, y hay certeza de la posición dentro de vía aérea, se debe continuar con el paso de la cánula de traqueostomía y completar el procedimiento como se ha indicado.

En caso de no poder ventilar y oxigenar el paciente a través de una máscara laríngea, se debe considerar la realización de cricotiroidotomía versus traqueostomía, dando prioridad a la última ya que minimiza el número de PGA y es un procedimiento definitivo comparado con la cricotiroidotomía, que es un procedimiento temporal y conlleva necesariamente a una traqueostomía.

\section{Lista de chequeo de insumos}

- Sistema de capnografía.

- Compresas para oclusión de fugas y control de aerosoles.

- Lidocaína $2 \%$ de uso parenteral

- Kit de traqueostomía percutánea

- Gel lubricante

- Aguja hipodérmica I4 Fr

- Jeringa de Iocc (conexión no roscada)

- Guía metálica

- Bisturí desechable

- Conductor de la guía

- Dilatador Rhino

- Dilatador de tamaño adecuado para la cánula

- Cánula de traqueostomía del tamaño indicado

- Endocánula (se requiere la del conector blanco para pacientes con ventilación mecánica)

- Correas fijadoras o cinta umbilical

- Gasas para protección de bordes del traqueostoma
- Manguera corrugada de conexión (extensión)

\section{Conclusiones}

Las intervenciones sobre la vía aérea generan riesgo de contagio del personal de salud al estar expuestos a los aerosoles provenientes de la vía aérea, y es por eso que la traqueostomía en pacientes COVID-I9 se convierte en uno de los procedimientos de mayor riesgo para el equipo quirúrgico.

Durante la actual pandemia, el personal sanitario debe tener especial cuidado en prevenir su contagio, para lo cual, como primera medida se debe elegir rigurosamente a los pacientes que realmente se beneficien de este procedimiento, y en los casos en que se considera necesaria, se debe contar con la protección de máximo nivel, según los protocolos institucionales para los EPP.

Si bien, en la mayoría de los casos la traqueostomía es un procedimiento programado que se realiza en pacientes con la vía aérea asegurada y conectados a ventilación mecánica, tiene el potencial de llevar rápidamente a una situación crítica, en la que no sólo se corre el riesgo de perder la vida del paciente, sino que se incrementa el riesgo de contagio al equipo quirúrgico al realizar medidas desesperadas que pueden romper las normas de bioseguridad indicadas en casos de pacientes infectados con SARS-CoV-2. Por lo tanto, es indispensable hacer un alistamiento previo al procedimiento, que incluya diferentes estrategias que favorezcan mantener el control durante todo el tiempo.

En los casos de emergencia en que no hay control de la vía aérea, se deben plantear alternativas a la intubación orotraqueal, como las máscaras laríngeas de última generación, para permitir la oxigenación y ventilación del paciente hasta que se realice la traqueostomía, evitando llegar a situaciones con medidas desesperadas que favorecen acciones que comprometen la bioseguridad del equipo quirúrgico. 
En ambas situaciones, es importante tener presente la necesidad de impedir fugas de la vía área, manteniendo estrategias como la relajación completa del paciente que evite los movimientos respiratorios o el reflejo tusígeno, y las barreras mecánicas con el uso de compresas húmedas alrededor de los implementos del kit y el traqueostoma, que pueden retener las gotas de aerosol con el fin de disminuir la probabilidad de contagio.

\section{Cumplimiento de normas éticas}

Consentimiento informado: Esta publicación es una revisión de la literatura y presenta la experiencia del grupo de Cirujanos de Trauma y Emergencias en la práctica de un procedimiento quirúrgico, sin poner en riesgo a los pacientes, por lo que no hay necesidad de un consentimiento informado.

Declaración de conflicto de intereses: Los autores no declaran ningún conflicto de interés.

Fuentes de financiación: Recursos propios de los autores.

\section{Referencias}

I. Kamps BS, Hoffmann C. COVID Reference. Esp. Edición 2020-I. Actualizado 07-04-2020. Hamburg: Steinhäuser Verlag ed. Disponible en: https://amedeo.com/CovidReferenceor_es.pdf

2. Asociación Colombiana de Cirugía. Recomendaciones para realización de traqueostomías y atención de los pacientes traqueostomizados en Colombia durante la pandemia COVID-I9. 2020;I-I3. Disponible en: https://ascolcirugia.org/ images/resources/PDF/comunicados/Recomendaciones_Traqueostomia.pdf

3. Asociación Colombiana de Cirugía. Recomendaciones para el manejo de los pacientes quirúrgicos urgentes durante la pandemia COVID-I9.
2020:I-I5. Disponible en: https://ascolcirugia. org/images/resources/PDF/comunicados/Recomendaciones_COVID-I9_CxGeneral_ACC_3003-2020.pdf

4. Asociación Española de Cirujanos. Documentos de posicionamiento y recomendaciones del grupo de trabajo cirugía - AEC-COVID-I9. 2020 [cited 2020 Mar 30]. Recomendaciones generales de actuación y organización básica a servicios de cirugía en zonas con alta afectación por la pandemia por COVID-I9 (SARS COV-2). Disponible en: https:// www.aecirujanos.es/Documentos-de-posicionamiento-y-recomendaciones-de-la-AEC-en-relacion-con-la-cirugia-y-COVIDı9_es_I_I52.html

5. Fundación Valle del Lili. Guía médica: Atención del paciente quirúrgico durante la pandemia de COVID-I9. Documentos Covid-I9. Abril io de 2020. Disponible en: https://valledellili.org/ coronavirus-covid-I9

6. Iftikhar IH, Teng S, Schimmel M, Duran C, Sardi A, Islam S. A network comparative meta-analysis of percutaneous dilatational tracheostomies using anatomic landmarks, bronchoscopic, and ultrasound guidance versus open surgical tracheostomy. Lung. 2019;197:267-75. https://doi. org/I0.IO07/s00408-019-00230-7

7. Tien HC, Chughtai T, Jogeklar A, Cooper $\mathrm{AB}$, Brenneman F. Elective and emergency surgery in patients with Severe Acute Respiratory Syndrome (SARS). Can J Surg. 2005;48;7I-4

8. Tay J, Li-Chung M, Shyang W. Surgical considerations for tracheostomy during the COVID pandemic. Lessons learned from the severe acute respiratory syndrome outbreak. JAMA Otolaryngol Head Neck Surg. Published Online: March 3I, 2020. https://doi.org/IO.IOOI/jamaoto.2020.0764

9. Balibrea JM, Badia JM, Rubio-Perez I, Antona EM, Alvarez-Peña E, García-Botella S, et al. Manejo quirúrgico de pacientes con infección por COVID-I9. Recomendaciones de la Asociación Española de Cirujanos. 2020. Disponible online el 4 de Abril de 2020. https://doi.org/IO.IoI6/j. ciresp.2020.03.00I 